\title{
RESEARCH ON DRUM WATER LEVEL CONTROL OF MARINE AUXILIARY BOILER BASED ON ADRC
}

Hui-bing Gan, Ph.D.

Bo Lv, Master.

College of Marine Engineering, Dalian Maritime University, Liaoning, Dalian, China

\begin{abstract}
SThe active disturbance rejection control (ADRC) exhibits strong robustness and adaptability in the presence of strong interference of a large class of uncertain systems. In order to better control the water level of the auxiliary boiler drum, this paper applies the ADRC to improve its control precision, robustness and timeliness. Firstly, the change of drum water level in a large oil tanker auxiliary boiler is analyzed and the dynamic equation is established. After the mathematic changes, the dynamic equation is transformed into a two-order system. Aiming at the characteristics of nonlinear, time-varying and strong disturbance of the boiler water level control system in marine auxiliary boiler, studying the ADRC that includes tracking differentiator (TD), extended state observer (ESO), nonlinear feedback (NLSEF) and disturbance compensation of four parts. The establishment of two kinds of two-order ADRC controller to control the drum level. Through the theoretical analysis and simulation experiment test, and comparing with the cascade control strategy, the experimental results show that the active disturbance rejection controller satisfies the steady state response index of the system, and has good timeliness and accuracy to the control of water level of the drum.
\end{abstract}

Keywords: ADRC; Robustness; Adaptability; Drum water level; Marine auxiliary boiler

\section{INTRODUCTION}

Drum water level is an important monitoring of boiler operation parameters, it is indirect reflects the boiler load and water balance, maintaining the water level is to maintain the drum important conditions for the safe operation of the boiler [1]. At present, most of the large oil tanker auxiliary boiler drum water level control system with high control accuracy is two impulses cascade control and three impulses cascade control. However, in the two impulses control or the three impulses control, there is a coupling between the feedforward loop of the steam flow and the feedback loop of the water supply valve due to the feedback error of the water level error, and the PID parameter will need to be retuning as the boiler conditions change. Digital method and advanced signal processing are usually incorporated into the PID to improve the PID controller's poor robustness, large overshoot and poor timeliness to improve its control performance.

Nowadays, many control strategies combined with PID applications have been proposed in the boiler. K.B. Lee et al. analyzed the dynamic characteristics of the power plants boiler water level of the drum, and applied fuzzy control in the drum water level control system [2, 3, 4]. Hamed Moradi et al. applied sliding mode control to the control of the power plants boiler drum water level system [5]. These control schemes described above have improved the control effect, but there are still some problems.

Active disturbance rejection control is a non-linear control strategy proposed by Jing-qing Han, China [6]. ADRC achieves real-time estimation and compensation of disturbance by establishing the extended state observer, thus avoiding the defects of integral action and improving the adaptability of the system. According to the direction and size of the state error to carry out nonlinear feedback to improve the stability and fastness of the system [7]. Based on the analysis of the uncertainty of the water level system, this paper designs two ADRC methods for the drum water level of the boiler. The simulation results show that the control method has the advantages of high precision and fast response. 


\section{ADRC OF DRUM WATER LEVEL}

ADRC is composed of tracking differentiator (TD), extended state observer (ESO), non-liner error feedback (NLSEF) and disturbance compensation [8].

The research on the water level of the drum of a large oil tanker boiler can obtain the dynamic equation of the change of drum water level [9]:

$$
T_{1} T_{2} \frac{d^{2} h}{d t^{2}}+T_{1} \frac{d h}{d t}=\left(T_{w} \frac{d u_{w}}{d t}+k_{w} u_{w}\right)-\left(T_{D} \frac{d u_{D}}{d t}+k_{D} u_{D}\right)
$$

In Eq. (1):

$T_{w} \frac{d u_{w}}{d t}+k_{w} u_{w} \quad$ - water disturbance;

$\left(T_{D} \frac{d u_{D}}{d t}+k_{D} u_{D}\right)-$ steam disturbance;

$h$ - drum water level;

$T$ - time constant.

The function of (1) can write as.

$\frac{d^{2} h}{d t^{2}}=\frac{1}{T_{1} T_{2}}\left[-T_{1} \frac{d h}{d t}+\left(T_{w} \frac{d u_{w}}{d t}+k_{w} u_{w}\right)-\left(T_{D} \frac{d u_{D}}{d t}+k_{D} u_{D}\right)\right]$

Using the two-order ADRC controller to study the water level of the drum.

Take the two-order object as an example, the dynamic equation of the controlled object can be described as:

$$
x^{2}=f(\dot{x}, x, w, t)+b u
$$

In Eq. (3):

$w$ - external disturbance;

$f$ - the generalized perturbation of the system;

$b$ - system compensation;

$u$ - system control input.

\section{ADRC CONTROLLER FOR DRUMWATER LEVEL}

In the ADRC system of the drum water level, all the uncertain factors (including the perturbation disturbance and external disturbance) of the drum water level are attributed to the generalized disturbance. Generalized disturbance quantities are estimated by ESO and through compensated by feedforward. The tracking signal of a given water level obtained by TD is compared with the estimated value of the system state variable obtained by ESO, and the control input signal of the system is obtained by NLSEF [8].

Drum water level ADRC controller algorithm is as follows. 1) Tracking Differentiator(TD)

$$
\left\{\begin{array}{l}
e=v_{1}-v \\
v_{1}=v_{1}+h v_{2} \\
v_{2}=v_{2}+h f_{\text {han }}\left(e, v_{2}, r_{0}, h_{0}\right)
\end{array}\right.
$$

In Eq. (4):

$v \quad$ - given signal;

$v_{1}$ - the tracking signal;

$v_{2}$ - the tracking speed;

$r_{0}$ - the speed factor;

$e \quad$ - the deviation of the tracking signal from the given signal;

$h$ - the sampling step;

$h_{0}$ - the filter factor;

$f_{\text {han }}$ - the optimal integrated control function [6].

The function of $f_{\text {han }}$ can be defined as

$$
f_{\text {han }}(e, v, r, h)=\left\{\begin{array}{l}
-r \operatorname{sign}(a),|a| \geq d \\
-r a / d,|a| \leq d
\end{array}\right.
$$

Where: $d=r h ; d_{0}=h d ; y=e+h v$

$$
\begin{gathered}
a_{0}=\sqrt{d^{2}+8 r|y|} \\
a=\left\{\begin{array}{l}
v+\operatorname{sign}(y)\left(a_{0}-d\right) / 2,|y|>d_{0} \\
v+y / d,|y| \leq d_{0}
\end{array}\right.
\end{gathered}
$$

2) Extended State Observer(ESO)

Aiming at two-order system with external disturbance and uncertainties.

$$
x^{2}=f(\dot{x}, x, t)+w(t)+b_{0} u(t)
$$

In Eq. (7):

$f(\dot{x}, x, t)-$ an unknown function;

$w(t)$ - the external disturbance;

$u(t)$ - control input;

$b_{0}$ - a constant.

order:

$$
\left\{\begin{array}{l}
x_{1}(t)=x(t) \\
x_{2}(t)=x(t) \\
x_{3}(t)=f(x, \dot{x}, t)+w(t)
\end{array}\right.
$$

The system (7) can be expressed as

$$
\left\{\begin{array}{l}
\dot{x}_{1}(t)=x_{2}(t) \\
\dot{x}_{2}(t)=x_{3}(t)+b_{0} u(t) \\
x_{3}(t)=\xi(t)
\end{array}\right.
$$

In Eq. (9):

$\xi(t)-$ an unknown function.

Establish a nonlinear system:

$$
\left\{\begin{array}{l}
\dot{z}_{1}(t)=z_{2}(t)-g_{1}\left(z_{1}(t)-x_{1}(t)\right) \\
\dot{z}_{2}(t)=z_{3}(t)-g_{2}\left(z_{1}(t)-x_{1}(t)\right)+b_{0} u(t) \\
\dot{z}_{3}(t)=-g_{3}\left(z_{1}(t)-x_{1}(t)\right)
\end{array}\right.
$$

In Eq. (10):

The function of $g_{1}\left(e_{1}\right), g_{2}\left(\mathrm{e}_{1}\right), g_{3}\left(e_{1}\right)$ is the proper nonlinear continuous function of the construction, according to (9) and (10), there are, 


$$
\left\{\begin{array}{l}
\dot{e}_{1}(t)=e_{2}(t)-g_{1}\left(e_{1}(t)\right) \\
\dot{e}_{2}(t)=e_{3}(t)-g_{2}\left(e_{1}(t)\right) \\
\dot{e}_{3}(t)=-\xi(t)-g_{3}\left(e_{1}(t)\right)
\end{array}\right.
$$

In Eq. (11):

$$
e_{i}(t)=z_{i}(t)-x_{i}(t)(i=1,2,3)
$$

For arbitrarily changed within a certain range $\xi(t)$, if and only when the nonlinear continuous function $g_{1}\left(e_{1}\right), g_{2}\left(\mathrm{e}_{1}\right), g_{3}\left(e_{1}\right)$ satisfies,

$$
e_{1} g_{i}\left(e_{1}\right)>0, \forall e_{1} \neq 0, g_{i}(0)=0(i=1,2,3)
$$

The system (12) is stable at the origin. Therefore, provided the appropriate nonlinear continuous function is constructed $g_{1}\left(e_{1}\right), g_{2}\left(\mathrm{e}_{2}\right), g_{3}\left(e_{3}\right)$, the state of the system (8) able to track the state of the system (9), namely,

$$
z_{1}(t) \rightarrow x_{1}(t), z_{2}(t) \rightarrow x_{2}(t), z_{3}(t) \rightarrow x_{3}(t)
$$

According to the definition in formula (8), $x_{3}(t)$ is equal to an unknown function $f(x, \dot{x}, t)$ plus external disturbance $w(t)$.As long as the function satisfies the condition of (12) $g_{1}\left(e_{1}\right), g_{2}\left(\mathrm{e}_{1}\right), g_{3}\left(e_{1}\right)$ satisfies the condition of $(12)$, then the output of the system $(10) z_{n+1}$ can be estimated $x_{n+1}(t)$ in real time, so the nonlinear system (10) is the ESO, ESO acquisition signal is derived from the input and output signals of the controlled object, For the $n$ order controlled object to establish the $n+1$ order estimation state system, the parameter selection can make the observer real-time accurate state of the controlled object estimate. Because of $x_{n+1}(t)$ is the extended state, it is equivalent to the acceleration of the system, which can reflect the dynamic, disturbance and uncertain information of the system. It's can be seen that the ESO is independent of the concrete form of the object description function [10].

To study the object of two-order uncertainty system.

$$
\left\{\begin{array}{l}
x_{1}^{(2)}=f\left(\dot{x}_{1}, x_{1}, w(t)+b_{0} u\right. \\
y=x_{1}
\end{array}\right.
$$

In Eq. (14):

Order $x_{2}=f\left(x_{1}, \dot{x}_{1}, w(t)\right.$ is a new state of system (14), the new system as

$$
\left\{\begin{array}{l}
x_{1}^{(2)}=x_{2}+b_{0} u \\
\dot{x}_{2}-\xi(t) \\
y=x_{1}
\end{array}\right.
$$

Where:

The function of $\xi(t)$ is derivative of the $x_{2}$, the unknown function. When $\xi(t)$ have the bounded, that is $|\xi(t)|<\xi_{0}$, the application $\mathrm{fal}$ function is used to construct the appropriate function, the ESO of the system (15) according to equation (10),

$$
\left\{\begin{array}{l}
e=z_{1}-y \\
\dot{z}_{1}=z_{2}-\beta_{01} e \\
\dot{z}_{2}=z_{3}-\beta_{02} \operatorname{fal}\left(e_{1}, a_{1}, \delta_{1}\right)+b_{0} u \\
\dot{z}_{3}=-\beta_{03} \operatorname{fal}\left(e_{2}, a_{2}, \delta_{2}\right)
\end{array}\right.
$$

Where:

$0<a_{1}<a_{2}<1, \beta_{01}, \beta_{02}, \beta_{03}$ is gain of the observer, the magnitude of derivative $\xi(t)$ varies with the change in the uncertainty of the system (14), and when the part changes rapidly, $\xi(t)$ is large. On the contrary, it is very small.

The fal function is a non-smooth function as follows:

$$
f a l(x, \alpha, \delta)=\left\{\begin{array}{l}
|x|^{\alpha} \operatorname{sign}(x),|x|>\delta \\
x / \delta^{1-\alpha},|x| \leq \delta
\end{array}\right.
$$

3) Non-liner Error Feedback (NLSEF)

$$
\left\{\begin{array}{l}
e_{1}=v_{1}-z_{1}, e_{2}=v_{2}-z_{2}, \\
u_{0}=\beta_{1} e_{1}+\beta_{2} e_{2}
\end{array}\right.
$$

Where:

The coefficient of $\beta_{1}, \beta_{2}$ is the gain factor.

4) Disturbance Compensation

$$
u=u_{0}-z_{3} / b_{0}
$$

Where:

The coefficient of $b_{0}$ is the compensation factor.

ADRC structure shown in Fig. 1. [6].

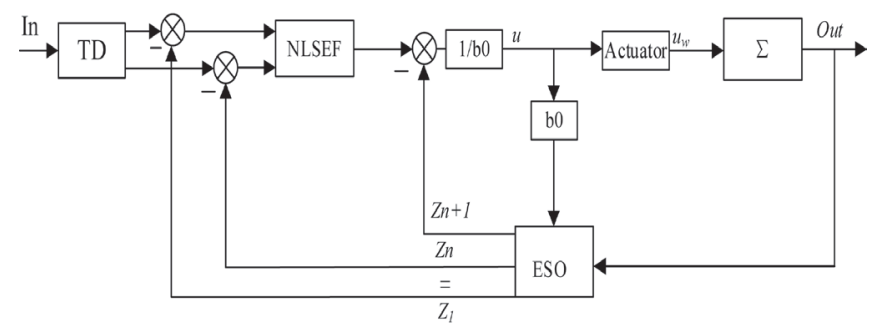

Fig. 1. Diagram of boiler water level control on $A D R C$

\section{PARAMETER TUNING OF THE ADRC CONTROLLER}

ADRC parameter tuning: First, for power parameters $a_{i}$, Usually in the ESO to take $a_{1}=0.5, a_{2}=0.25$ In the nonlinear feedback $0<a_{1} \leq 1.0, a_{2} \geq 1.0$, usually to take $a_{1}=0.75$ or 0.5 , $a_{2}=1.25$ or 1.5 .

The parameter $r$ of the TD is related to the excessive process time $T_{0}$ :

$$
r=\frac{4\left(x_{1}-x_{0}\right)}{T_{0}^{2}}
$$


Where the coefficient of $x_{1}$ is the set value of TD, $v$ is the input value, and $\mathrm{x}_{01}$ is the initial value of $v . r$ to determine the tracking signal and speed, in order to suppress overshoot, generally taken $r<1$.

The ADRC controller's gain factor $\beta_{1}, \beta_{2}$ corresponds to the proportional derivative gain of the PID controller, and the compensation factor $b_{0}$ is equivalent to the integral gain of the PID controller [15]. To adjust the parameters in the extended state observer $\beta_{01}, \beta_{02}, \beta_{03}$, the system by the sampling time to determine the three parameters. Where $\beta_{03}$ is the most critical, the greater the value, the system lag is smaller, but $\beta_{03}$ too large will cause oscillation, so in the adjustment process, the first adjustment $\beta_{03}$ until the observer tracking effect is better, and then fine tune $\beta_{1}$ and $\beta_{2}$ to Enhance the performance of the observer [11]. $b_{0}$ is the compensation of the disturbance, it can refer to the PID control strategy in the differential and integral items to be adjusted.

\section{SIMULATION OF CONTROL PROCESS OF DRUM WATER LEVEL ADRC}

Fig. 2. is ADRC cascade three impulse working principle [12].

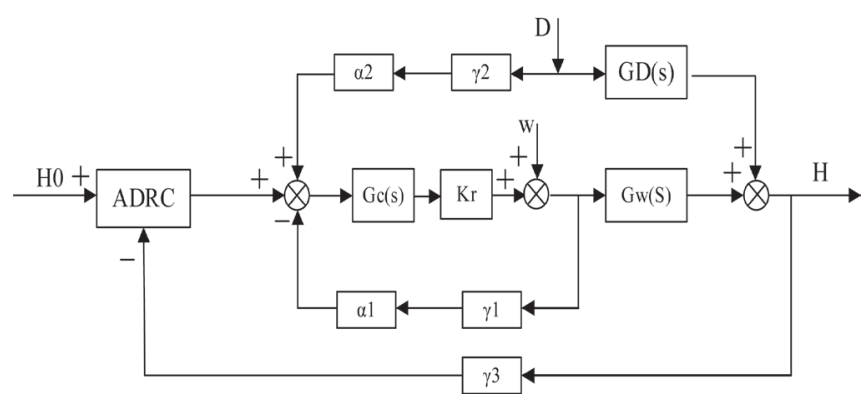

Fig. 2. The working principle of ADRC with cascade three impulses

Fig. 2., $H, H_{0}$ is the water level and its given value respectively. $w$ is the water flow and $D$ is the steam flow, $G_{w}(s), G_{D}(s)$ they are steam flow and the water flow of the transfer function of drum level.

Where:

$$
\begin{gathered}
G_{w}(s)=\frac{0.037}{s(30 s+1)}, G_{D}(s)=\frac{3.6}{15 s+1}-\frac{0.037}{s}, \\
\gamma_{1}=\gamma_{2}=0.083, \alpha_{1}=\alpha_{2}=0.21, \gamma_{3}=0.033, K_{r}=20 .
\end{gathered}
$$

At the same time on the PID controller parameter tuning. $K_{p 1}=0.5, K_{i 1}=0.0025, K_{d 1}=8, K_{p 2}=1, K_{i 1}=0.2, K_{d 2}=0$

Design two kinds of ADRC, and compare the performance of both.

\section{1) ADRC without Tracking Differentiator}

By simulation debugging, get a better set of parameters is: ESO: $\beta_{01}=6, \beta_{02}=12, \beta_{03}=8$, ;NLSEF:, $\beta_{1}=0,05, \beta_{2}=0.8$. disturbance compensation: b01 $=0.0023$. In the simulation experiment, take the sampling step $h=0.2 s$. The secondary PID parameter is: $K_{p}=1.45, K_{i}=0.0055, K_{d}=0$.

In the simulation experiment, the simulation time $t=800 \mathrm{~s}$ is added to the $35 \%$ feed water flow disturbance, the simulation time $t=1200 \mathrm{~s}$ is added to the $30 \%$ steam flow disturbed. The control goal is to stabilize the drum level to 10 per unit. The corresponding unit step response curve is shown in Fig. 3.

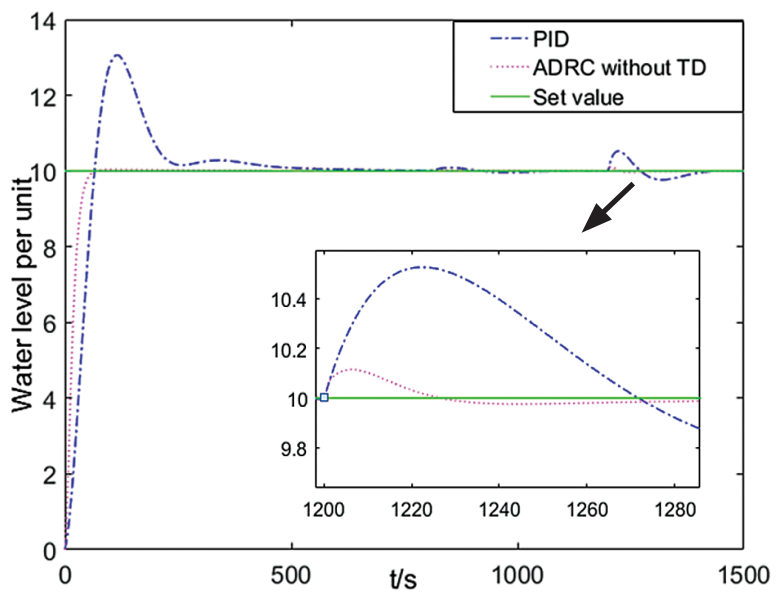

Fig. 3. The cascade three impulses of ADRC without tracking differentiator

As can be seen from Fig. 3., the ADR step response without TD is fast and no overshoot, the system takes a short time to reach steady state, the PID control overshoot is large and the timeliness is poor. When adding water flow perturbation has little effect on PID and ADRC without TD has no change in steady state. When adding the steam flow perturbations, the contribution of the control flow to the control system has apparently overshoots. It can be seen from enlarged picture that the ADRC control system without TD is obviously smaller than the PID control system in transition time and overshoot.

2) ADRC with tracking differentiator

By simulation debugging, get a better set of parameters is: TD: $r=5, h=0.2$; ESO: $\beta_{01}=100, \beta_{02}=50, \beta_{03}=10$; NLSEF: $\beta_{1}=5$, $\beta_{01}=25$; disturbance compensation: $b_{0}=0.0023$. The secondary loop of the PID parameters as above. As shown in Fig. 4.

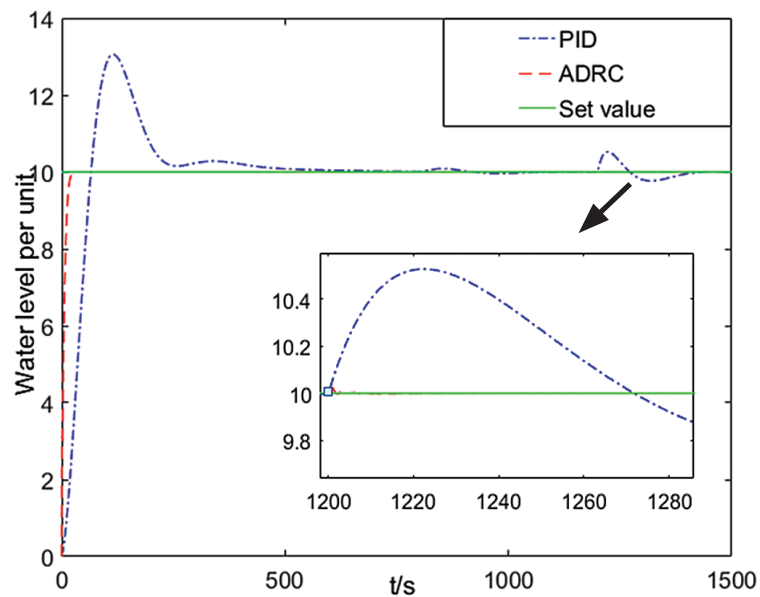

Fig. 4. The cascade three impulses of ADRC

It can be seen from Fig. 4. that the response speed of ADRC is fast and there is no overshoot. When adding water flow disturbances have little effect on the two controllers, when adding steam flow disturbances have almost no effect on ADRC, it can be seen from the enlarged graph that overshoot 
is very small, the entire controller showed a good adaptability and timeliness.

3) Experimental Comparison of Two Kinds of Active Disturbance Rejection Controllers

The simulation results are shown in Fig. 5.

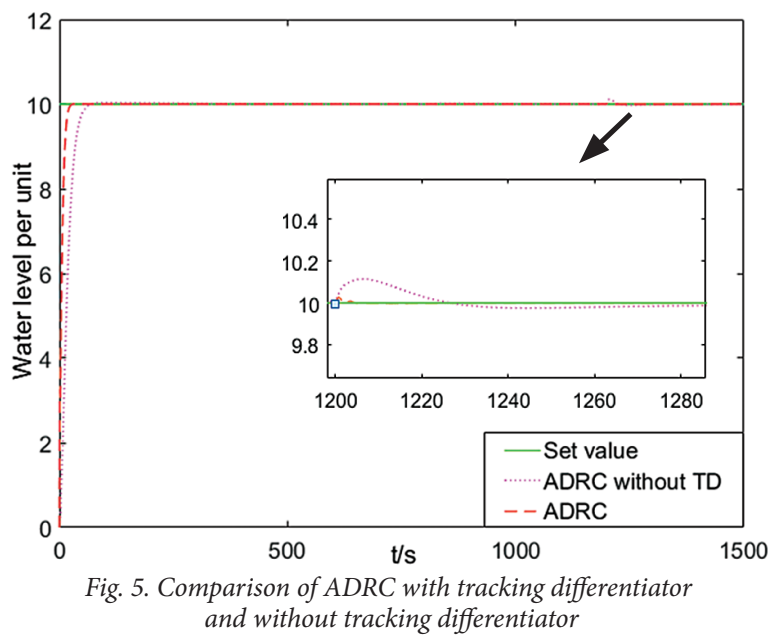

As can be seen from Figure 5, the ADRC with TD rises faster and reaches the steady state faster than the ADRC without TD as the step response curve is rising. However, there has jitter as the ADRC with TD is on the rise, while the ADRC without TD is more stable during the ascent. When adding water flow disturbances have no effect on these two ADRC controllers. When adding the disturbance of steam flow, the ADRC control system without TD has larger the overshoot than ADRC with TD. The more robust of the ADRC control system with TD, and the superior control performance of the two controllers compared to PID control, showing a very strong robustness.

To change the condition, when the time constant of the controlled object model increases $67 \%$, the proportional coefficient increases 35\%, the controlled object becomes

$$
G_{w}(s)=\frac{0.05}{s(50 s+1)} .
$$

The simulation results are shown in Fig. 6.

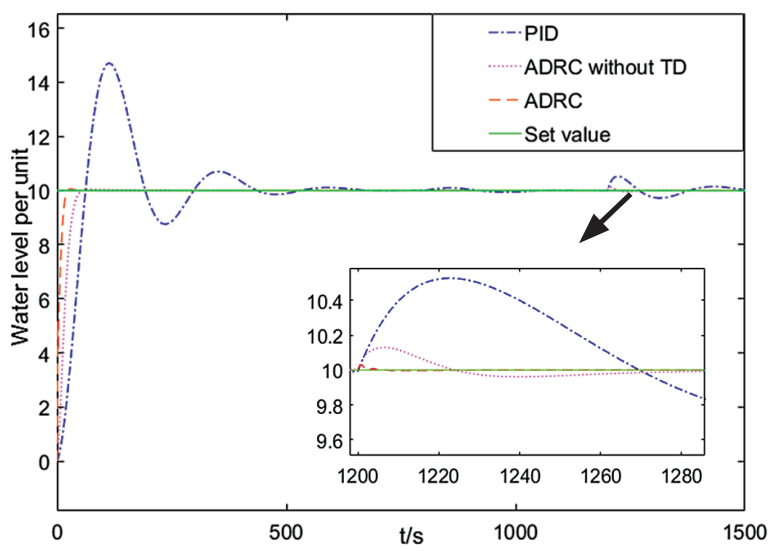

Fig. 6. ADRC under Changing Conditions
The simulation results are not changed, as shown in Fig. 7.

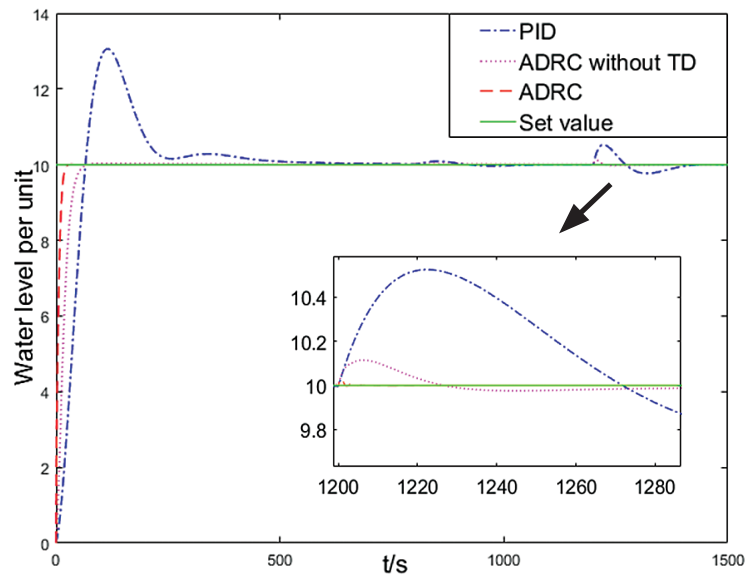

Fig. 7. ADRC without changing conditions

From Fig. 6. and Fig. 7. we can see that when the transfer function of feedwater flow changes, the overshoot of PID control becomes larger and the time for the system to reach steady state becomes longer. The ADRC controller with TD and the ADRC controller without TD adjust the feed water flow disturbance suppression ability is strong, no overshoot. To establish two kinds of ADRC control system can restrain the influence of a variety of uncertainties in the system well, with high control precision, fast response, strong adaptability, good robustness and so on.

\section{CONCLUSION}

This paper analyzes the dynamic change of the drum level of a large oil tanker and establishes the two-order model. By applying the ADRC control theory to the drum level control system of a large oil tanker auxiliary boiler, two kinds of ADRC controllers are designed and implemented. One is the traditional ADRC controller, and the other is to remove the TD simplified ADRC controller. Firstly, two kinds of drum water level cascade three-impulse ADRC algorithm are designed according to the non-linear, time-varying and strong interference of the drum water level system after obtaining the mathematical model of the water level of the three impulse drums. The performance of the control algorithm is tested based on the experimental model. It can be seen from the simulation results that the ADRC system can suppress the influence of various uncertainties in the system, and has the advantages of high control precision, fast response speed. Although the controller has shown the strong robustness and adaptability and achieves the expected results, it is necessary to further study in the future application due to the complexity of the control model and many parameters that need to be adjusted in the controller. 


\section{ACKNOWLEDGMENTS}

The project is supported by "Liaoning Provincial Natural Science Foundation of China" (NO.201602071), also supported by "the Fundamental Research Funds for the Central Universities" (NO.3132016316). The authors also gratefully acknowledge the helpful comments and suggestions of the reviewers, which have improved the presentation.

\section{REFERENCES}

1. Hong-jun Liu, Pu Han, Dong-feng Wang.: Simulation of Boiler Drum Water Level System DMC-PID Cascade Control. Journal of System Simulation, 2004 (03): 450-453.

2. Xiao Feng.: Fuzzy self-adapting PID control of drum water level in a power plant, IFAC Proceedings Volumes, Volume 40, Issue 8, 2007, pp. 77-83.

3. Nan-hua Yu, Wen-tong Ma, Ming Su.: Application of adaptive Grey predictor based algorithm to boiler drum level control, Energy Conversion and Management, Volume 47, Issue 18, 2006, pp 2999-3007.

4. Jing-tao Zhang, Wei Wang, Tian-you Chai, et al.: Intelligent Fuzzy Control of the Power Plant Boiler Water Level, IFAC Proceedings Volumes, Volume 32, Issue 2, 1999, pp. 7241-7246.

5. Hamed Moradi, Majid Saffar-Avval, Firooz Bakhtiari-Nejad.: Sliding mode control of drum water level in an industrial boiler unit with time varying parameters: A comparison with - robust control approach, Journal of Process Control, Volume 22, Issue 10, 2012, pp. 1844-1855.

6. Jing-qing Han.: Auto disturbance rejection control technique. Frontier Science, 2007, 1(1): 24-31.

7. Hai-sheng Li.: Research on Parameter Tuning and Optimization of Active Disturbance Rejection Controller, Control Engineering. 2004,11(5): 419-423.

8. Hong Chen, Jian Zeng, Guang-jun Wang.: Active Disturbance Rejection Control of Steam Generator Water Level Proceedings of the CSEE, 2010, (32): 103-107.

9. 9. Ju-yuan Jiang, Li-jing Li.: The research on the new boiler water level automatic control system. Mechanic Automation and Control Engineering (MACE), 2011 Second International Conference on, 2011.

10. Jing-qing Han.: Auto disturbance rejection control technique. Beijing: National Defense Industry Press, 2009: 255-262,280286 (in Chinese).
11. Yong-chun Fang, Hui Shen, Xiu-yun Sun, Xu Zhang,Bin Xian.: Control of Active Disturbance Rejection Control of Unmanned Helicopter. Control Theory and Applications, 2014, 31(02): 238-243.

12. Chang-mei Hu, Jun Ren.: Research and application of linear ADRC on cascade three-impulse control of drum water level. Electric Power, 2014, 47 (12): 28-31.

13. Lin Fei, Hu Sun.: A new type of extended state observer with a measurement noise system. Control Theory and Applications. 2005, 22(6): 995-998

14. Zhi-qiang Gao.: Scaling and bandwidth-parameterization based controller tuning Proceeding of the American Control Conference. United States: IEEE, 2003: 4989-4996.

15. Simna Surendran, Vimal Kumar.: Neural Network Based PI Controller Parameter Calculation on a Boiler Drum Level System, Procedia Technology, Volume 24, 2016, pp. 1616-1622

16. Hai-sheng Li.: Research on Parameter Tuning and Optimization of Active Disturbance Rejection Controller.Control Engineering. 2004, 11(5): 419-423.

17. Jing-qing Han.: Active disturbance rejection controller and its application. Control and Decision, 1998, (01): 19-23.

18. Yuan-qing Xia, Li Dai, Meng-yin Fu, et al.: Application of active disturbance rejection control in tank gun control system, Journal of the Franklin Institute, Volume 351, Issue 4, 2014, pp. 2299-2314.

19. Fei Xia, Hao Zhang, Gui Zhang, et al.: Image Processing in the Monitor of Boiler Drum Water Level on Power Plant, Energy Procedia, Volume 17, 2012, pp. 266-272.

20. Jing-qing Han.: Auto disturbance rejection control technique Frontier Science, 2007, 1(1): 24-31.

21. Sun, K., L.P. Huang and Y. Mei.: A nonlinear auto-disturbance rejection control for matrix converter fed induction motor drive system. Transactions of China Electro technical Society 2007, 22(12), 39-45.

22. Jing-qing Han, Wen-ge Zhang.: Active Disturbance Rejection Control for Large Delay Systems. Control and Decision, 1999, (04): 67-71.

23. Xin Leng, Qi-dan Zhu.: Multi-model Predictive Control of Boiler Water Level for Marine Boiler Based on Radial Basis Function Neural Network Dynamic Compensation. Journal of Jilin University (Engineering and Technology Edition) 2011, 41(05): 1450-1455. 
24. Hang Yi, Luo ZW, Svinin M, et al.: Extended state observer technique for control of robot systems. The Proc. of the 4th IEEE World Congress on Intelligent Control and Automation. Shanghai, 2002.

25. Y. Huang, H. Wan, J.L. Song, Analysis and Design for Third Order Nonlinear Continuous Extended Status Observer. Proceeding of 19th Chinese Control Congress. Hong Kong, China, 2000: 677-681.

26. Min Xu, Shao-yuan Li, Wen-jian Cai, Cascade generalized predictive control strategy for boiler drum level, ISA Transactions, Volume 44, Issue 3, 2005, pp. 399-411.

\section{CONTACT WITH THE AUTHOR}

Hui-bing Gan, Ph. D. e-mail:ghbzq@dlmu.edu.cn Faculty of Marine Engineering College Dalian Maritime University

Dalian

China 